\title{
Spatial and Temporal Power Allocation for MISO Systems with Delayed Feedback
}

\author{
Venkata Sreekanta Annapureddy and Srikrishna Bhashyam \\ Department of Electrical Engineering \\ Indian Institute of Technology Madras \\ Chennai 600036, India \\ Phone: 91-44-22574439, Fax: 91-44-22570120 \\ E-mail:vannapu2@uiuc.edu,skrishna@ee.iitm.ac.in
}

\begin{abstract}
We determine the minimum outage probability of multiple-input single-output (MISO) channels in the presence of delayed feedback. Channel state information at the transmitter (CSIT) is a delayed version of the perfect channel state information available at the receiver (CSIR). Under a short-term power constraint, we determine: (a) the outage probability for beamforming with imperfect CSIT (BF-IC) analytically, and (b) the optimal spatial power allocation (OSPA) scheme that minimizes outage numerically. Results show that, for delayed CSIT, BF-IC is close to optimal for low SNR and uniform spatial power allocation (USPA) is close to optimal at high SNR. Similarly, under a long-term power constraint, we determine the outage probability for BF-IC with temporal power control and compare it with USPA with temporal power control.
\end{abstract}

\section{INTRODUCTION}

The minimum outage probability of multiple-input single-output (MISO) channels with perfect channel state information at the receiver (CSIR) and no channel state information at the transmitter (CSIT) is derived in [1]. Results for perfect CSIT and CSIR are presented in [2]. Minimum outage transmission under a short term power constraint for two cases of imperfect CSIT, mean feedback and covariance feedback, has been recently studied in [3], [4]. In [5], maximizing mutual information in the presence of channel estimation error has been studied. Under a long-term power constraint, minimum outage probability with temporal power control for quantized CSIT and delayed CSIT have been studied in [6] and [7] respectively.

In this paper, we analyze minimum outage transmission over MISO channels using the delayed feedback model in [7] under both short-term and long-term power constraints. Under a short-term power constraint (i. e.,

V.S. Annapureddy was supported in part by NSF under CCF0431088 at the University of Illinois, through a subcontract with University of Wisconsin. when transmit power is constant for each transmission interval), the outage probability for beamforming with imperfect CSIT (BF-IC) is determined analytically. The minimum outage transmission strategy, optimal spatial power allocation (OSPA), is also obtained. OSPA involves beamforming along the spatial modes and optimal power allocation across the spatial modes. Results for uniform spatial power allocation (USPA) [1] are also compared. The results show that BF-IC is very close to OSPA for low SNR while USPA is close to OSPA for high SNR. Since OSPA does not provide significant gain at any SNR, compared to the best of BF-IC and USPA, the cross-over SNR at which USPA becomes better than BF-IC is important. We present the equation to determine this cross-over SNR and solve it numerically.

Under a long-term power constraint [2], we outline the optimal spatial and temporal power allocation strategy. The outage probability of the BF-IC scheme with temporal power control is also determined. Results from [7] on USPA with temporal power control are also compared. Again, we show that even with temporal power control, BF-IC is better at low SNR while USPA is better at high SNR. The cross-over SNR when USPA becomes better than BF-IC can be determined.

\section{SyStem MODEL}

The MISO system with $M$ transmit antennas and one receive antenna is, as usual, modeled as

$$
y=\mathbf{h}^{H} \mathbf{x}+z,
$$

where $\mathbf{h} \sim \mathcal{C N}(\mathbf{0}, \mathbf{I})$ is a $M \times 1$ independent, identically distributed (i.i.d) and zero-mean circularly symmetric complex Gaussian channel vector, $\mathbf{x}$ is a $M \times 1$ channel input vector and $z$ is zero-mean unit-variance additive white Gaussian noise (AWGN). Using the Gaussian channel vector model, we can relate the old channel and 
the actual channel as [7].

$$
\mathbf{h}=\rho \mathbf{h}_{\text {old }}+\sqrt{1-\rho^{2}} \mathbf{w}
$$

where $\mathbf{h}_{\text {old }}$ is the delayed CSIT, $\rho$ is a correlation coefficient and $\mathbf{w}$ is $\mathcal{C N}(\mathbf{0}, \mathbf{I})$.

\section{Short-Term Power Constraint}

Assuming a short-term power constraint, the mutual information is given by

$$
I\left(\mathbf{x} ; y / \mathbf{h}, \mathbf{h}_{\text {old }}\right)=\log \left(1+P \mathbf{h}^{H} \mathbf{Q h}\right)
$$

where $\mathbf{Q}$ is the input covariance matrix such that $\operatorname{Tr}(\mathbf{Q})=1$ and $P$ is the transmit power. Consider the two extreme cases: zero feedback $(\rho=0)$ and instantaneous feedback $(\rho=1)$. For $\rho=0$, Uniform Spatial Power Allocation (USPA) is optimal [1], i.e., we have

$$
\begin{gathered}
\mathbf{Q}=\frac{\mathbf{I}}{M}, \\
P_{\text {out } \mathbf{U S P A}}(M, R, P)=\Gamma_{M}\left(\frac{e^{R}-1}{P / M}\right) .
\end{gathered}
$$

For $\rho=1$, beamforming is optimal [2], i.e.,

$$
\begin{gathered}
\mathbf{Q}=\frac{\mathbf{h h}^{H}}{\mathbf{h}^{H} \mathbf{h}}, \\
P_{\text {out } \mathbf{B F}}(M, R, P, \rho=1)=\Gamma_{M}\left(\frac{e^{R}-1}{P}\right) .
\end{gathered}
$$

Outage performance for $\rho=1$ is $10 \log _{10} \mathrm{M} \mathrm{dB}$ better than the performance for $\rho=0$. We consider the case of $0<\rho<1$.

\section{A. Beamforming using imperfect CSIT (BF-IC)}

In this case, beamforming is performed using the delayed CSIT assuming that it is the actual channel. Therefore, we have

$$
\mathbf{Q}=\frac{\mathbf{h}_{\text {old }} \mathbf{h}_{\text {old }}^{H}}{\mathbf{h}_{\text {old }}^{H} \mathbf{h}_{\text {old }}} .
$$

The mutual information can be simplified as

$$
I\left(\mathbf{x} ; y / \mathbf{h}, \mathbf{h}_{\text {old }}\right)=\log \left(1+P \frac{\left(1-\rho^{2}\right)}{2} A\right),
$$

where $A=\left|\sqrt{2 \delta}+\sqrt{2} \frac{\mathbf{w}^{H} \mathbf{h}_{o l d}}{\sqrt{\gamma}}\right|^{2}, \delta=2 \mu \gamma, \mu=\frac{\rho^{2}}{1-\rho^{2}}$ and $\gamma=\mathbf{h}_{\text {old }}^{H} \mathbf{h}_{\text {old }}$ is the feedback SNR. Note that A, given $\gamma$ is a non-central chi-square (nc- $\chi^{2}$ ) random variable with two degrees of freedom and parameter $\delta$. Therefore we have the following expression for the outage probability for a given $\gamma$.

$$
\operatorname{Pr}(\text { outage } / \gamma)=F_{\left(\mathrm{nc}-\chi^{2}, 2, \delta\right)}(2 \beta),
$$

where $\beta=\frac{e^{R}-1}{P}(\mu+1)$, and $F_{\left(\mathrm{nc}-\chi^{2}, 2, \delta\right)}(\cdot)$ is the $\mathrm{CDF}$ of a non-central chi-square random variable with two degrees of freedom and parameter $\delta$. The overall probability of outage can be simplified as

$$
\begin{aligned}
& P_{\text {out } \mathbf{B F - I C}}(M, R, P, \rho)=\int_{0}^{\infty} f_{\Gamma}(\gamma) \operatorname{Pr}(\text { outage } / \gamma) d \gamma \\
& =\frac{1}{(1+\mu)^{M-1}} \sum_{i=0}^{M-1}\left(\begin{array}{c}
(M-1) \\
i
\end{array}\right) \mu^{i} \Gamma_{(i+1)}\left(\frac{e^{R}-1}{P}\right) .
\end{aligned}
$$

The derivation of equation (11) is shown in the appendix. The asymptotic diversity gain at infinite SNR, defined as

$$
d=-\lim _{\mathrm{SNR} \rightarrow \infty} \frac{\log P_{\text {out }}}{\log S N R},
$$

can be quantified. From (11), using the approximation $\Gamma_{M}(x) \simeq \frac{x^{M}}{M !}$ for very small $x$, we can show that the asymptotic diversity gain of the BF-IC scheme is 1 for imperfect CSIT, i.e.,

$$
\text { Diversity Gain } d=\left\{\begin{array}{ll}
1 & \text { for } 0 \leq \rho<1 \\
M & \text { for } \rho=1
\end{array} .\right.
$$

This result can be explained intuitively as follows. At very high SNR, the outage probability is dominated by the error in the CSIT rather than channel being in deep fade. However, for USPA, the asymptotic diversity gain is $M$ independent of $\rho$. Therefore, USPA is always better than BF-IC at high SNR. The cross-over SNR $S N R_{\text {cross }}(\rho, R, M)$ can be obtained by equating the outage probabilities of the two schemes: (5) and (11). Although there is no closed form expression for crossover SNR, it can be computed numerically. By comparing the operating SNR with the cross-over SNR, one can switch between BF-IC and USPA.

\section{B. Optimal Spatial Power Allocation (OSPA)}

Now, we find the optimal spatial power allocation strategy that minimizes the outage probability. OSPA allocates a fraction $\lambda$ of the power along the spatial mode corresponding to the imperfect CSIT with the remaining power being equally distributed among the other orthogonal spatial modes.

The outage probability is minimized by minimizing $\operatorname{Pr}($ outage $/ \gamma)$ for each $\gamma \cdot \operatorname{Pr}($ outage $/ \gamma)$ is given by

$$
\operatorname{Pr}(\text { outage } / \gamma)=\operatorname{Pr}\left(\mathbf{h}^{H} \mathbf{Q h}<\frac{e^{R}-1}{P}\right)
$$

Using (2), the outage probability given by (14) is equivalent to the outage probability of a MISO channel with an AWGN variance of $\left(1-\rho^{2}\right)$ and a mean feedback $\frac{\rho}{\sqrt{1-\rho^{2}}} \mathbf{h}_{\text {old }}$. The outage probability of a MISO 
channel with mean feedback is minimized without any loss of generality by minimizing over the fraction of the power spent in the direction of the mean feedback [3], [4]. Rest of the power is spent equally in the M1 orthogonal beams. We derive an analytical expression for $P_{\text {out }}(\gamma, \lambda)$, the outage probability as a function of $\gamma$, the feedback SNR, and $\lambda$.

$$
\begin{aligned}
& P_{\text {out }}(\gamma, \lambda)=\operatorname{Pr}\left(\lambda A+\frac{1-\lambda}{M-1} B<2 \beta\right) \\
= & \int_{0}^{\frac{2 \beta}{\lambda}} f_{A}(a) F_{B}\left(\frac{(2 \beta-\lambda a)(M-1)}{1-\lambda}\right) d a
\end{aligned}
$$

where $\mathrm{A}$ is non-central chi-square distributed with 2 degrees of freedom and non-centrality parameter $\delta=$ $2 \mu \gamma, \mathrm{B}$ is central chi-square distributed with $2(\mathrm{M}-1)$ degrees of freedom. Then, the optimal $\lambda$ should be obtained for each $\gamma$.

$$
\begin{aligned}
& P_{\text {out OSPA }}(M, R, P, \rho)= \\
& \qquad \int_{0}^{\infty} f_{\Gamma}(\gamma) P_{\text {out }}\left(\gamma, \lambda_{\text {opt }}(\gamma)\right) d \gamma
\end{aligned}
$$

where $\lambda_{\text {opt }}(\gamma)$ is the solution of

$$
\frac{\partial P_{\text {out }}(\gamma, \lambda)}{\partial \lambda}=0
$$

Using (15), (17) can be simplified as

$$
\begin{aligned}
\int_{0}^{\frac{2 \beta}{\lambda}} f_{A}(a) \exp & \left(\frac{(M-1) \lambda a}{2(1-\lambda)}\right) \\
& (2 \beta-\lambda a)^{(M-2)}(2 \beta-a) d a=0
\end{aligned}
$$

Since no closed form expression for $\lambda_{\text {opt }}$ is possible as a function of $\gamma, \lambda_{o p t}$ is determined numerically. For $\gamma$ close to zero, the mean feedback is zero and so, it is optimal to split equal power in all the spatial modes, i. e., $\lambda_{\text {opt }}(\gamma=0)=\frac{1}{M}$. For higher values of $\gamma$, it is optimal to spend all the power in the direction of the imperfect CSIT $\left(\lambda_{\text {opt }}(\gamma \rightarrow \infty)=1\right)$.

\section{Results \& Observations}

Fig 1 shows the performance of USPA and BF-IC for different values of $\rho$ and $M=2$. Fig 2 compares the outage probability of BF-IC and USPA with OSPA for $\rho=0.9$ and $M=2$ and Fig 3 shows the outage performance for different number of transmit antennas for $\rho=0.999$. The cross-over SNR is plotted as a function of $\rho$ in Fig 4 and Fig 5 shows $\lambda_{o p t}(\gamma)$ as a function $\gamma$ for the OSPA scheme. All the figures are generated for $R=2$ nats $/ \mathrm{s} / \mathrm{Hz}$.

- BF-IC with $\rho=0$ gives SISO performance. $P_{\text {ou } t \text { BF-IC }}(M, R, P, \rho=0)=\Gamma_{1}\left(\frac{e^{R}-1}{P}\right)$
- BF-IC is better at lower SNR's and worse at high SNR's when compared to USPA for any $\rho<1$

- It can be seen from Fig 3 that BF-IC gives an asymptotic diversity gain of only 1 , independent of M. With BF-IC, diversity gain increases with SNR initially, reaches a maximum and then decreases with SNR and asymptotically reaches 1 for infinite SNR, unlike the case with USPA, where it increases monotonically with SNR.

- Cross-over SNR $S N R_{\text {cross }}(\rho, R, M)$ is a monotonically increasing function of $\rho$. Therefore, higher the value of $\rho$, wider the range of SNR over which BF-IC is better than USPA.

- In practice, instead of using OSPA, it would be sufficient to switch between BF-IC and USPA depending on the average SNR, because (1) Performance of BF-IC is very close to OSPA at low SNR and the performance of USPA is very close to OSPA at high SNR, (2) OSPA is computationally complex since it requires computing $\lambda_{\text {opt }}(\gamma)$ for each channel realization and (3) Any small error in the value of $\rho$ used to obtain the OSPA scheme will degrade performance significantly.

\section{LONG-Term Power Constraint}

We relax the short-term power constraint and consider a long-term power constraint i.e. transmitted power can be varied and is allocated dynamically with an average power constraint.

$$
\int_{0}^{\infty} f_{\Gamma}(\gamma) p(\gamma) d \gamma=1
$$

Achieving minimum outage probability involves power allocation in both spatial and temporal domains.

\section{A. Optimal Spatio-Temporal Power Allocation}

Optimal spatio-temporal power allocation is equivalent to OSPA + temporal power control. The problem of minimizing outage probability with temporal power control can be formulated as

$$
\min _{p(\gamma)} \int_{0}^{\infty} f_{\Gamma}(\gamma) P_{\text {out }}\left(\gamma, p(\gamma), \lambda_{\text {opt }}(\gamma, p(\gamma))\right) d \gamma
$$

subject to the power constraint (19), where $\lambda_{\text {opt }}(\gamma, p(\gamma))$ is the solution of

$$
\frac{\partial P_{\text {out }}(\gamma, p(\gamma), \lambda)}{\partial \lambda}=0
$$

Similar to $(15), P_{\text {out }}(\gamma, p(\gamma), \lambda)$ is given by

$$
P_{\text {out }}(\gamma, p(\gamma), \lambda)=\operatorname{Pr}\left(\lambda A+\frac{1-\lambda}{M-1} B<\frac{2 \beta}{p(\gamma)}\right)
$$


However, finding optimal $p(\gamma)$ and the corresponding $\lambda_{\text {opt }}(\gamma, p(\gamma))$ is computationally intensive, since we do not have closed form expression for $\lambda_{o p t}(\gamma, p(\gamma))$. Therefore, based on the intuition from the results for the short-term power constraint, BF-IC with temporal power control and USPA with temporal power control are considered. The outage probability of USPA with optimal temporal power control was calculated in [7].

\section{B. BF-IC with Temporal Power Control}

We evaluate the outage probability of BF-IC $(\lambda=1)$ with temporal power control numerically.

$$
P_{\text {out }}(\gamma, p(\gamma))=\operatorname{Pr}\left(A<\frac{2 \beta}{p(\gamma)}\right)=F_{\left(\mathrm{nc}-\chi^{2}, 2, \delta\right)}\left(\frac{2 \beta}{p(\gamma)}\right)
$$

With $\lambda=1$, (20) is modified to

$$
\min _{p(\gamma)} \int_{0}^{\infty} f_{\Gamma}(\gamma) F_{\left(\mathrm{nc}-\chi^{2}, 2, \delta\right)}\left(\frac{2 \beta}{p(\gamma)}\right) d \gamma
$$

Using calculus of variations, we arrive at the following expression for the power control function that minimizes the outage probability with BF-IC.

$$
k=\left(\frac{2 \beta}{p^{2}(\gamma)}\right) f_{\left(\mathrm{nc}-\chi^{2}, 2, \delta\right)}\left(\frac{2 \beta}{p(\gamma)}\right)
$$

where $\mathrm{k}$ is chosen so that $p(\gamma)$ thus obtained satisfies the power constraint (19) and is non-negative. This can be solved numerically.

\section{Results \& Observations}

Fig 2 shows the performance of BF-IC and USPA with and without temporal power control. As in the case of the short-term power constraint, temporal power control with BF-IC is better for low SNR and temporal power control with USPA is better at high SNR. The trends in the results are similar in both cases and the cross-over SNR is slightly lower with temporal power control.

\section{SUMMARY}

For a short-term power constraint, we derive an analytic expression for the outage probability of beamforming using imperfect CSIT (BF-IC) and compare it with that of uniform spatial power allocation (USPA). We also compute the outage probability for optimal spatial power allocation (OSPA) numerically. Results show that BF-IC is close to optimal at low SNR, while USPA is close to optimal at high SNR, i.e, OSPA does not improve the outage probability significantly compared to switching between BF-IC and USPA depending on the average SNR. For a long-term power constraint, i.e., with temporal power control, we determine the outage probability of BF-IC numerically and compare it with USPA. Again, we observe that BF-IC is better at low SNR, while USPA is better at high SNR.

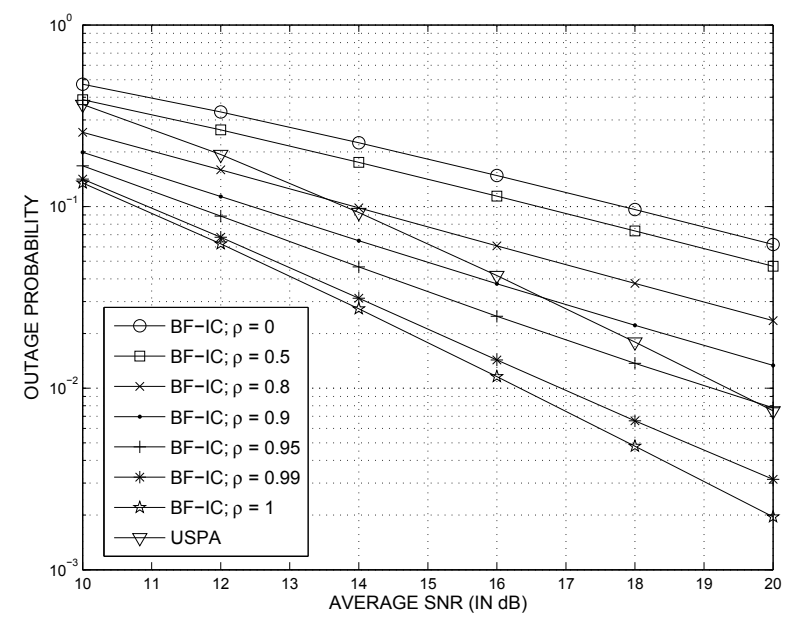

Fig. 1. Outage probabilities for Beamforming using imperfect CSIT (BF-IC) for various values of $\rho$, and uniform spatial power allocation (USPA) for $M=2$ and $R=2$ nats $/ \mathrm{s} / \mathrm{Hz}$.

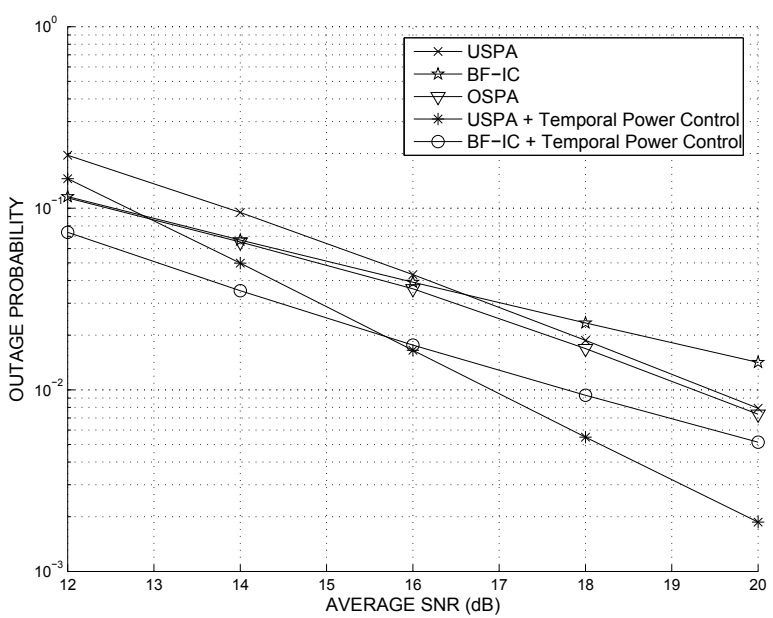

Fig. 2. Outage Probabilities for uniform spatial power allocation (USPA) and beamforming using imperfect CSIT (BF-IC) with and without temporal power control, and optimal spatial power allocation (OSPA) for $\rho=0.9 ; M=2$ and $R=2$ nats $/ \mathrm{s} / \mathrm{Hz}$.

\section{APPENDIX \\ DERIVATION OF EQUATION (11)}

The cdf of a non-central $\chi^{2}$ variable is given by

$F_{\left(\mathrm{nc}-\chi^{2}, 2 M, \delta\right)}(y)=\sum_{k=0}^{\infty} \frac{\left(\frac{\delta}{2}\right)^{k} e^{-\frac{\delta}{2}}}{k !} F_{\chi^{2}, 2 M+2 k}(y)$ 


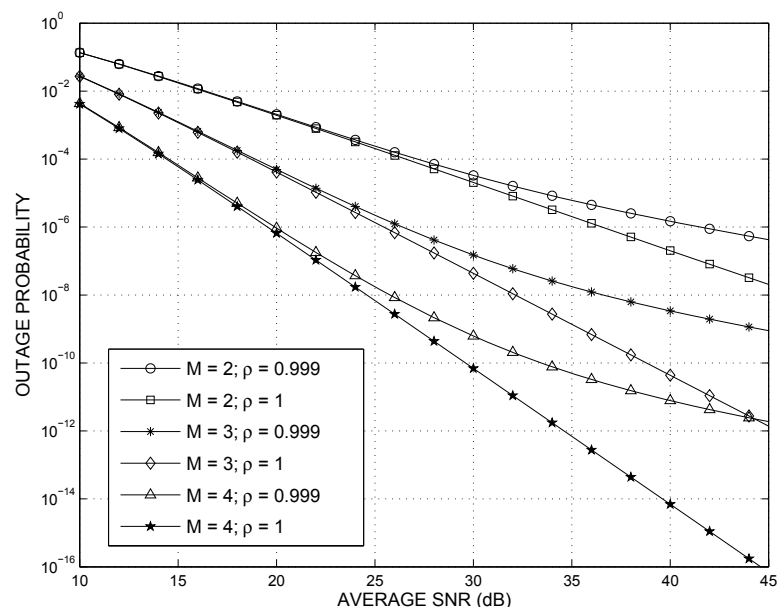

Fig. 3. Outage probability with BF-IC for various values of $M$ for $\rho=0.999$ and beamforming for $\rho=1$ and $R=2$ nats $/ \mathrm{s} / \mathrm{Hz}$

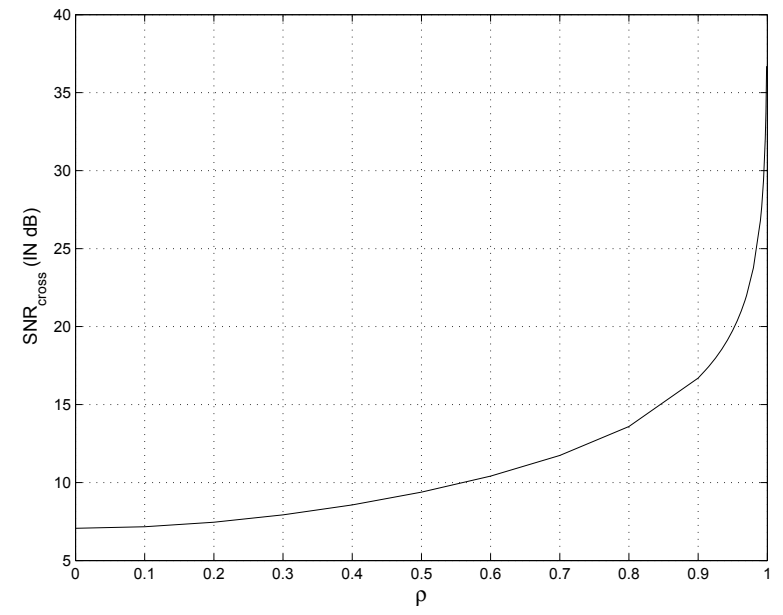

Fig. 4. Cross-over SNR $S N R_{\text {cross }}$ as a function of $\rho$ for $M=2$ and $R=2$ nats $/ \mathrm{s} / \mathrm{Hz}$.

where $F_{\chi^{2}, 2 M+2 k}(y)$ is the cdf of a central $\chi^{2}$ random variable with $2 M+2 k$ degrees of freedom. Using (26), $P_{\text {out } \mathbf{B F}-\mathrm{IC}}(M, R, P, \rho)$ is simplified as

$$
\frac{1}{(1+\mu)^{M}} \int_{0}^{\beta} e^{-x} g(x) d x
$$

$$
\text { where, } \begin{aligned}
g(x) & =\sum_{k=0}^{\infty} \frac{\left(\begin{array}{c}
M+k-1 \\
k
\end{array}\right)}{k !}\left(\frac{\mu x}{1+\mu}\right)^{k} \\
& =e^{\left(\frac{\mu x}{1+\mu}\right)} \sum_{i=0}^{M-1} \frac{\left(\begin{array}{c}
M-1 \\
i
\end{array}\right)}{i !}\left(\frac{\mu x}{1+\mu}\right)^{i}
\end{aligned}
$$

After substituting (28) in (27) and making a change of variable: $x=(1+\mu) y$, we get (11)

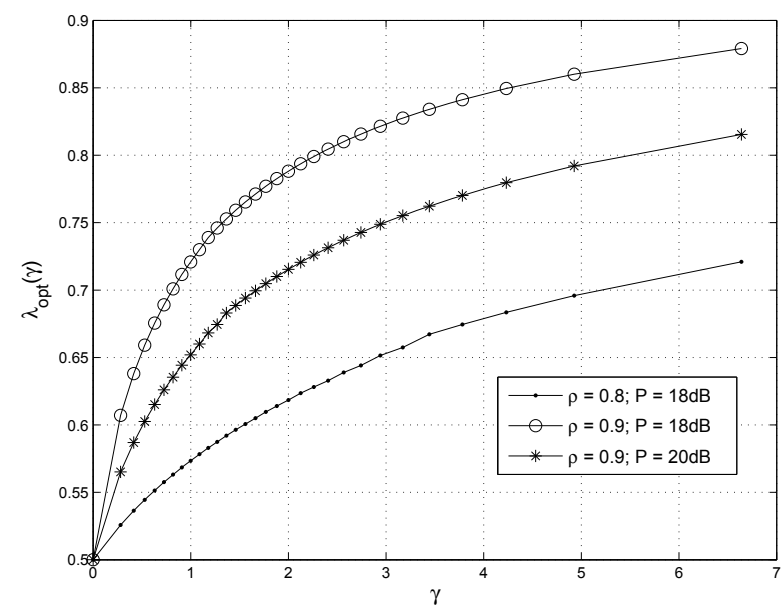

Fig. 5. Fraction of the power in the direction of imperfect CSIT $\lambda_{\text {opt }}(\gamma)$ for different values of $\rho$ and $P ; M=2$ and $R=2$ nats/s/Hz.

\section{APPENDIX \\ DERIVATION OF EQUATION (15)}

With out any loss of generality, $\mathbf{h}_{\text {old }}$ can be expressed as $\mathbf{h}_{\text {old }}=\mathbf{V d}$, where $\mathbf{d}$ is a $M \times 1$ vector with only one non-zero element and $\mathbf{V}$ is an unitary matrix.

$$
\mathbf{d}=\left[\begin{array}{llll}
\sqrt{\gamma} & 0 & \ldots & 0
\end{array}\right]^{T}, \text { where } \gamma=\left\|\mathbf{h}_{\text {old }}\right\|^{2}
$$

$\widetilde{\mathbf{Q}}=\mathbf{V}^{H} \mathbf{Q V}=\operatorname{Diag}\left[\begin{array}{llll}\lambda & \lambda_{0} & \ldots & \lambda_{0}\end{array}\right] \cdot \operatorname{Tr}(\mathbf{Q})=1 \Rightarrow \lambda_{0}=$ $\frac{1-\lambda}{M-1}$. Denoting $\mathbf{w}$ by $\mathbf{V g}, \mathbf{h}^{H} \mathbf{Q h}$ can be expressed as

$$
\left(\rho \mathbf{d}+\sqrt{1-\rho^{2}} \mathbf{g}\right)^{H} \widetilde{\mathbf{Q}}\left(\rho \mathbf{d}+\sqrt{1-\rho^{2}} \mathbf{g}\right)
$$

Substituting (30) in (14) and simplifying, we get (15).

\section{REFERENCES}

[1] E. Telatar, "Capacity of multi-antenna Gaussian channels," European Trans. Telecomm., Vol. 10, No. 6, pp 585-596, Nov. 1999.

[2] E. Biglieri, G. Caire, and G. Taricco, "Limiting Performance of block-fading channels with multiple antennas," IEEE Trans. on Info. Theory, Vol. 47, No. 4, pp. 1273-1289, May 2001.

[3] Y. Xie, C. N. Georghiades and A. Arapostathis, "Minimum Outage Probability Transmission With Imperfect Feedback for MISO Fading Channels", IEEE Trans. on Wireless Comm., Vol. 4, No. 3, pp. 1084-1091, May 2005.

[4] A.L. Moustakas, S.H. Simon, "Optimizing multiple-input singleoutput (MISO) communication systems with general Gaussian channels: nontrivial covariance and nonzero mean", IEEE Trans. on Info. Theory, Vol. 49, No. 10, pp. 2770-2780, Oct. 2003.

[5] T. Yoo and A. Goldsmith, "Capacity and power allocation for fading MIMO channels with channel estimation error," IEEE Trans. on Info. Theory, Vol. 52, No. 5, pp. 2203-2214, May 2006.

[6] S. Bhashyam, A. Sabharwal and B. Aazhang, "Feedback gain in multiple antenna systems," IEEE Trans. on Comm., Vol. 50, No. 5, pp 785-798, May 2002.

[7] D. V. Marathe and S. Bhashyam, "Power Control for Multiantenna Gaussian Channels with Delayed Feedback", Proceedings of the 39th Asilomar Conference on Signals, Systems and Computers, pp. 1598-1602, Oct-Nov 2005. 